\title{
Science with ESO's Multi-conjugate Adaptive-optics Demonstrator - MAD
}

\author{
Jorge Melnick, Enrico Marchetti, and Paola Amico \\ European Southern Observatory, Karl Schwarzschild Str. 2, D85748-Garching, Germany
}

\begin{abstract}
ESO's Multi-conjugate Adaptive-optics Demonstrator (MAD) was a prototype designed and built to demonstrate wide-field adaptive optics science on large telescopes. The outstanding results obtained during commissioning and guaranteed time observations (GTO) prompted ESO to issue and open call to the community for 23 science demonstration (SD) observing nights distributed in three runs (in order to provide access to the summer and winter skies). Thus, in total MAD was used for science for 33 nights including the 10 nights of GTO time. To date, 19 articles in refereed journals (including one in Nature) have been published based fully or partially on MAD data. To the best of our knowledge, these are not only the first, but also the only scientific publications from MCAO instruments world-wide to date (at least in Astronomy). The scientific impact of these publications, as measured by the $h$-index, is comparable to that of other AO instruments on the VLT, although over the years these instruments have been allocated many more nights than MAD. In this contribution we present an overview of the scientific results from MAD and a more detailed discussion of the most cited papers.
\end{abstract}

Keywords: Multi-conjugate adaptive optics, MCAO, MAD, Scientific Results, ESO

\section{INTRODUCTION}

Multi-Conjugate Adaptive Optics instruments (MCAO) are essential for 'very large' ground-based telescopes. Very large means mirror diameters larger than $\sim 25 \mathrm{~m}$ for conventional telescopes, but larger than only a few meters for Solar telescopes. In either case, however, the price to pay for not having MCAO is to be condemned to a very small field of view (FOV). Thus, although there are (expensive) ways around this problem (e.g. MOAO), the capacity to image relevant areas of sky at (or near) the diffraction limit of these extremely large telescopes is among the most important scientific drivers for building such telescopes on the ground.

The most ambitious of these projects was the 100m OverWhelmingly Large (OWL) telescope concept that was developed by ESO in the first lustrum of the $\mathrm{XXI}^{\text {st }}$ century AD. Mostly for reasons of risk and cost, the project morphed into the $42 \mathrm{~m}$-E-ELT, which eventually converged to the $39 \mathrm{~m}$ European Extremely Large Telescope (E-ELT) program that was recently endorsed by the ESO Council (in June 2012). A critical milestone for the OWL was to demonstrate the feasibility of building and operating MCAO instruments on large telescopes. This gave rise to the MAD project, whose aim was to demonstrate the scientific potential of MACAO imagers at the VLT. MAD was enthusiastically endorsed in 2001 by ESO's Scientific-Technical Committee (STC), which also recommended that the instrument be constructed on a fast track.

In practical terms, this recommendation implied that MAD had to be developed using, as much as possible, existing spares and leftovers from previous ESO (AO) projects. Thus, for example, the deformable mirrors (DM) and the real time computer (RTC) came from MACAO spares; the detector for the IR camera (CAMCAO) was an existing engineering-grade $2048 \times 2048$ Hawaii-II device*; the control system was as simple as possible while strictly adhering to the ESO/VLT standards; filter exchanges had to be done manually at the telescope, etc. MAD was a prototype intended to demonstrate the technology and not - at all - as common user instrument, so these 'shortcuts' were not only necessary to cut time and cost, but also fully consistent with the mission of the instrument.

A ground-breaking innovative feature of MAD was the so-called Layer Oriented mode (LO), which used up to 8 pyramid WFS thus allowing to combine the light of up to 8 AO stars and therefore to use stars significantly

Address all correspondence to jmelnick@eso.org

${ }^{*}$ This choice meant that the camera had to be mounted on a moving stage to cover the full 2-arcmin FOV of MAD 
fainter than the Shack-Hartmann mode (SH; also called Star Oriented mode - SO). Detailed descriptions of MAD, its history, and its technical characteristics have been presented at previous SPIE meetings and a good set of bibliographic references can be found in the papers by Marchetti et al. ${ }^{1}$ and Arcidiacono et al. ${ }^{2}$

The on-sky demonstration of MAD was so successful, that the community, via the STC, urged ESO to open the instrument to a wider audience. Thus, MAD was offered for 23 Science Demonstration nights divided in three runs (November 2007, January 2008, and August 2008). Due to operational constrains, the instrument could only be offered in SH mode, which imposed a stringent magnitude limit $(V \sim 13)$ for the WFS stars. This, compounded with the geometry of the 3-star asterisms required to have reasonably uniform Strehl ratios over the FOV of MAD (see Figure 1), implied that there was a limited number of targets that could be observed with full MCAO correction in the southern skies.
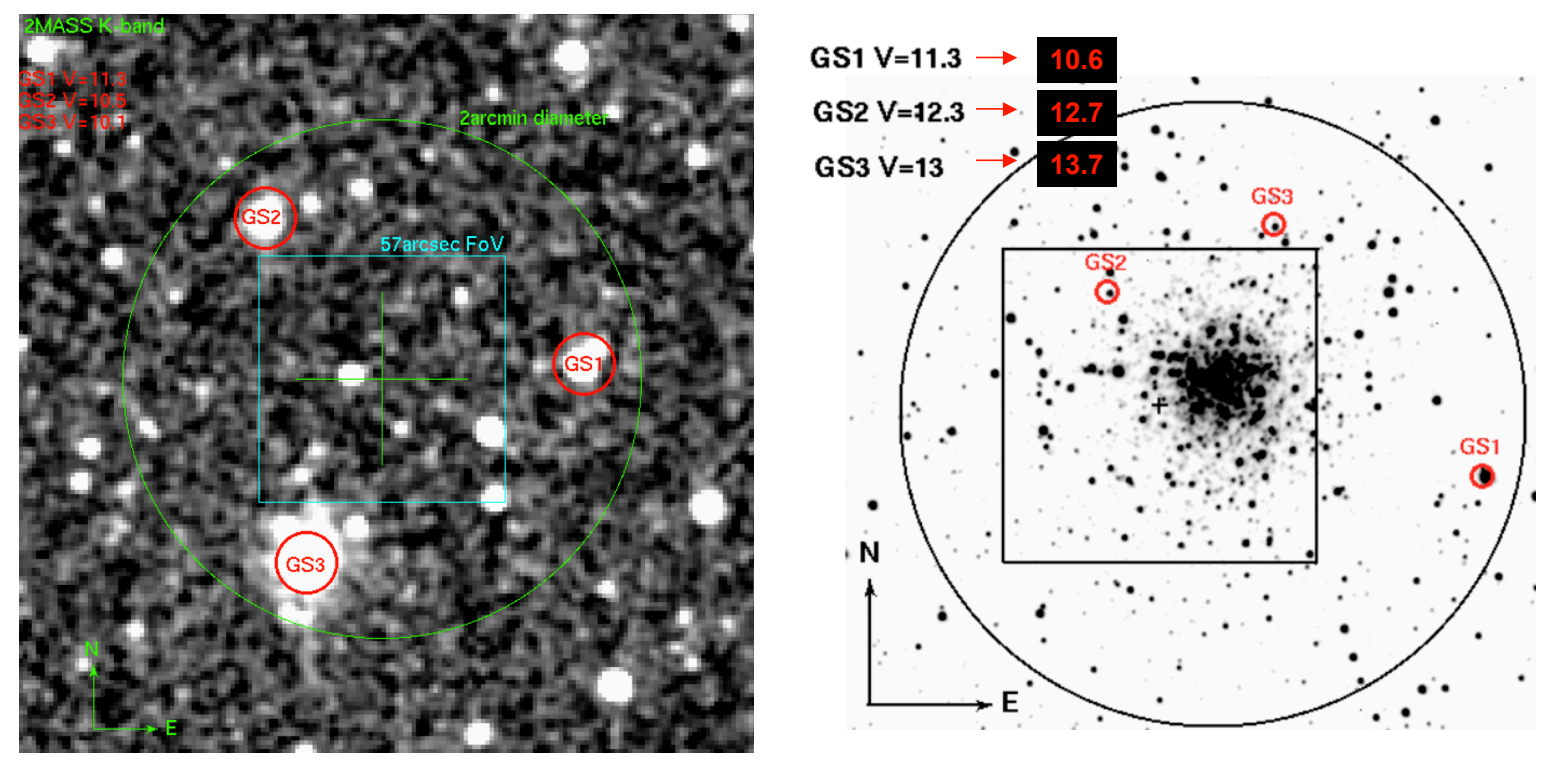

Figure 1. Left: An ideal asterism for MAD in SH mode: stars of similar magnitudes and equilateral geometry with the target at the center. Right: A difficult asterism: the magnitudes are quite different and the geometry is skewed.

In this contribution we present an overview of the most relevant scientific results produced by MAD, and a detailed discussion of the papers with the highest scientific impact as measured by the number of citations in refereed journals taken from the arXiv/astro-ph without filtering-out self-references, or references to technical rather than scientific issues.

\section{MAD SCIENTIFIC RESULTS}

\subsection{Operations}

Figure 2 presents a comparison of the overheads of MAD compared to those of other instruments at the VLT, with and without AO. The figure shows that the overheads of MAD are not much larger than those of other AO assisted instruments on the VLT. At first sight this may seem surprising considering the extra-complexity of the MAD target acquisition procedure involving three WFS stars in the SH mode, and that the AO calibration and optimization was performed on-sky before every observation, plus the additional overheads manual filter changes. In hindsight, however, MAD was operated by a 'black-belt' team including several of the people that actually developed the instrument. 


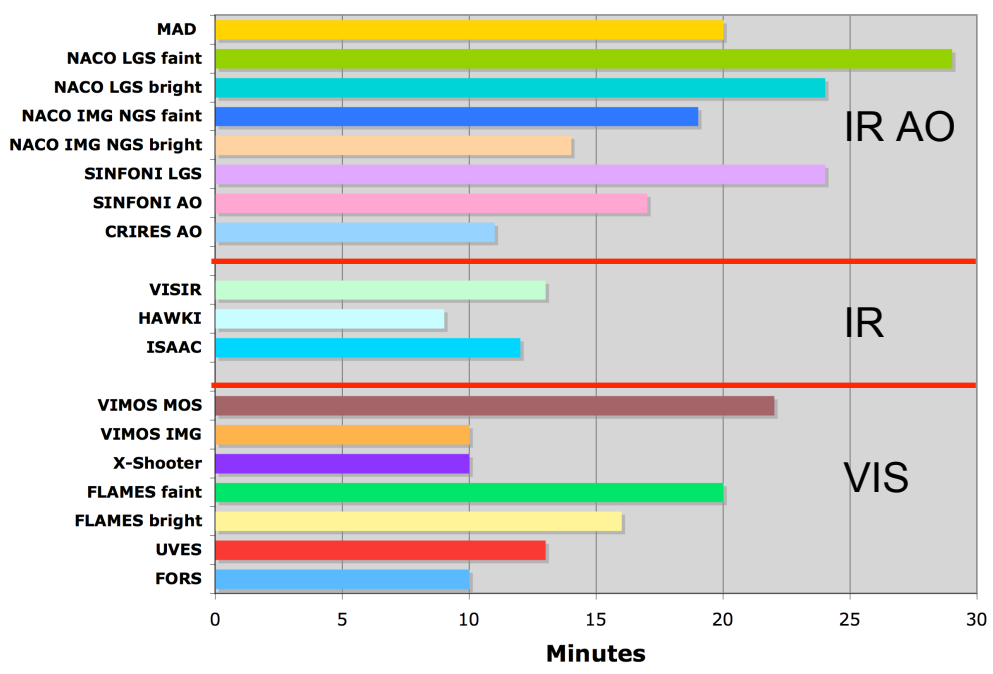

Figure 2. Acquisition times for VLT instruments including 6 minutes to preset the telescopes.

\subsection{Scientific Impact}

A total of 23 proposals were received to the widely advertised call for MAD SD programs. The scientific impact of the publications that resulted from these and the GTO observations (10 nights in total for CAMCAO and LO), as measured by the $h$-index (Figure 3 - left panel), is actually comparable to that of other AO instruments on the VLT, which however have observed for more nights. The right panel of Figure 3 lists the 19 refereed publications based on MAD data ordered by number of citations in refereed journals as given by the arXiv/astro-ph.

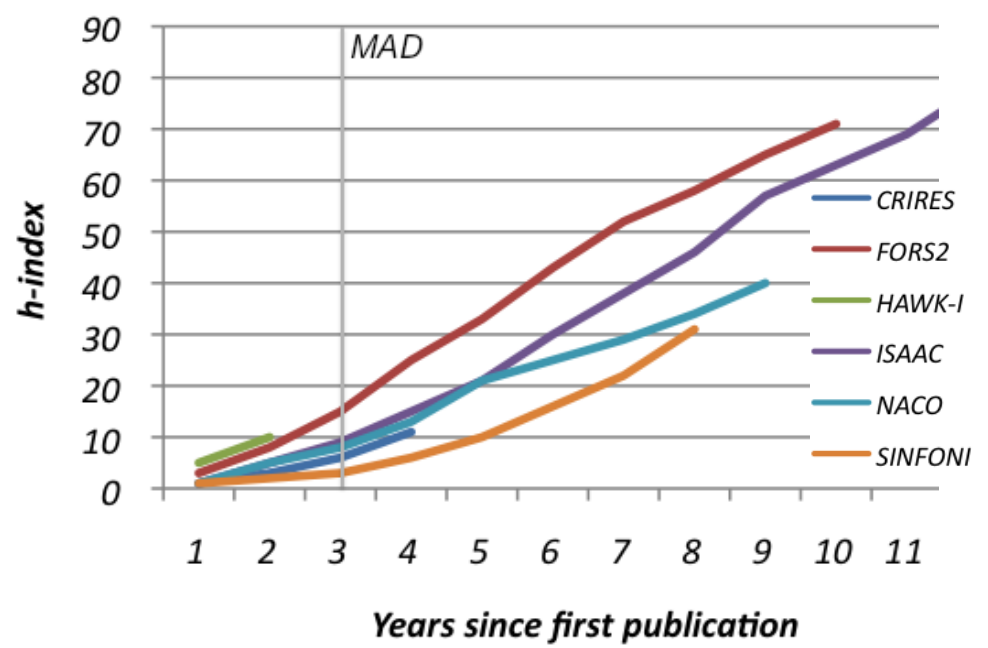

\begin{tabular}{|c|c|c|c|c|c|c|}
\hline Rank & First Author & Subject & Journal & Year & Citations & Mode \\
\hline 1 & Crowther & Massive Stars & MNRAS & 2010 & 48 & SH \\
\hline 2 & Ferraro & Globular Clusters & Nature & 2008 & 40 & SH \\
\hline 3 & Moretti & Globular Clusters & A\&A & 2009 & 19 & LO \\
\hline 4 & Campbelll & Massive Stars & MNRAS & 2010 & 16 & SH \\
\hline 4 & Sana & Massive Stars & A\&A & 2010 & 16 & SH \\
\hline 6 & Bouy & Massive Stars & A\&A & 2009 & 13 & SH \\
\hline 7 & Momany & Open Clusters & MNRAS & 2008 & 12 & SH \\
\hline 7 & Bouy & Massive Stars & A\&A & 2008 & 12 & SH \\
\hline 9 & Ferraro & Globular Clusters & ApJ & 2010 & 10 & SH \\
\hline 10 & Bono & Globular Clusters & ApJ & 2010 & 6 & SH \\
\hline 10 & Bouy & Massive Stars & A\&A & 2009 & 6 & SH \\
\hline 12 & Gullieuszik & Dwarf Galaxies & A\&A Let. & 2008 & 5 & LO \\
\hline 13 & Falomo & Blazar Jets & A\&A & 2009 & 4 & LO \\
\hline 14 & Mignani & Neutron Stars & A\&A RN & 2008 & 2 & LO \\
\hline 14 & Ortolani & Globular Clusters & ApJ & 2011 & 2 & SH \\
\hline 14 & Fiorentino & Magellanic Clouds & A\&A & 2011 & 2 & SH \\
\hline 17 & Luizzo & Blazar Jets & A\&A & 2011 & 0 & LO \\
\hline 18 & Rochau & Massive Stars & MNRAS & 2011 & 0 & SH \\
\hline 19 & Meyer & Astrometry & A\&A & 2011 & 0 & LO \\
\hline
\end{tabular}

Figure 3. Left: Scientific impact as measured by the $h$-index as a function of time in years since the first publication. The $h$-index of MAD about 3.5 years after the first publication is 10, which is comparable to that of other AO instruments at the VLT that observed for more nights. Right: The 19 publications based on MAD data arranged by number of citations in refereed journals as given by the arXiv.

The majority of the publications deal with stellar populations in clusters, ranging from substellar and planetary mass objects in Orion, ${ }^{3,4}$ to supermassive hyper-giants in 30 Doradus. ${ }^{5,6} \mathrm{~A}$ number of papers deal with more 'normal' stellar populations in young clusters, ${ }^{7,8}$ in Globular clusters, ${ }^{9,10,11,12,13,14}$ in old open clusters, ${ }^{15}$ 
and in the field of nearby dwarf galaxies. ${ }^{16,17}$ As expected from the requirements on the asterisms for $\mathrm{SH}$ mode discussed above, only a few extragalactic targets (besides local dwarfs) where successfully observed by MAD, mostly in LO mode. ${ }^{18,19}$ However, these observations targeted at the compact jets of BL Lac objects, actually used the wide-field capabilities of MAD only to search for suitable WFS stars. An extragalactic MAD deep field was attempted in SH mode, but the weather conditions did not allow us to reach the depth required to characterize the morphology of early-type galaxies as a function of redshift.

A particularly successful observation with MAD, which has not (yet) led to refereed publications, was the detection of changes in Jupiter's high atmosphere. Still, the spectacular MAD image of Jupiter (shown below) was published in newspapers and magazines around the world.

\subsection{MCAO top-level scientific requirements}

The top-level scientific requirements for an MCAO imaging system can probably be summarized as follows: (1) stable and uniform PSF; (2) accurate photometry with a large dynamic range; and (3) high astrometric precision, all three at or near the diffraction limit of the telescope and over a FOV substantially wider than that of single conjugate $\mathrm{AO}$ (SCAO) instruments.

Many of the references cited in the previous section show Strehl maps of the MAD FOV, and comment upon the uniformity and stability of the PSF, so the first of the top-level requirements was fully met by MAD. A particularly striking demonstration of the uniform PSF and high Strehl is presented in Figure 4 that shows an image of Jupiter taken in the Ks-band using Io and Europa as WFS 'stars'. The image is diffraction limited over the $1^{\prime}-\mathrm{FOV}{ }^{20}$
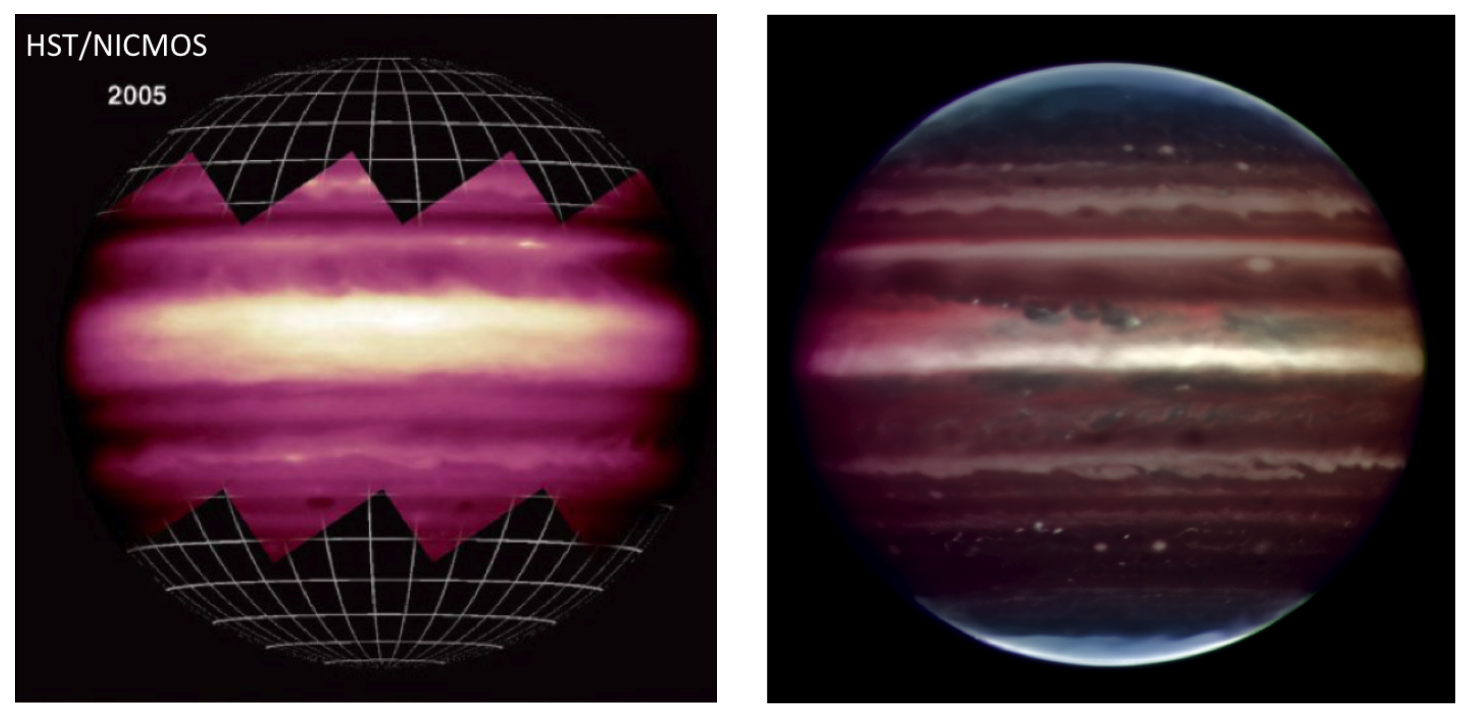

Figure 4. Comparison between a NICMOS K-band image of Jupiter taken in 2005 (left), with a Ks image taken with MAD in 2008 (right). The shift of the band of Jupiter's tropospheric equatorial haze is immediately apparent in the MAD image.

Several papers also discuss the photometric precision of MAD over its $11+$ magnitudes dynamic range. An example of the photometric resolving power of MAD is presented in Figure 5. The left panel shows an H-band image of the vicinity of the bright star $\lambda$-OriA illustrating the dynamical range and resolution of MAD. ${ }^{4}$ The authors were looking for sub-stellar companions in the $\lambda$-Orionis cluster. In a previous paper dealing with the Trapezium cluster in Orion they remark that '...MAD can easily reach $\Delta M=6$ mag at (separations of) 0.6 ", 0.5 ", and 0.4 " in $\mathrm{J}, \mathrm{H}$, and Ks respectively', demonstrating the potential of MAD for doing accurate photometry in crowded fields.

However, triaging field stars often requires something more than photometry. HST demonstrated for globular clusters that astrometry can be a very powerful tool to separate the clusters from the field. Nevertheless, another insidious effect comes into play for low-latitude and Bulge clusters: differential (foreground) extinction, and here 
is where near-IR photometry becomes extremely useful. As it turned out, MAD delivered a similar FOV and spatial resolution in the near-IR as the HST Advanced Camera for Surveys (ACS) at visible wavelengths, and several of the MAD programs exploited this wonderful synergy to observe globular clusters in crowded lowlatitude fields.

The astrometric precision of MAD was investigated both in $\mathrm{LO}$ mode ${ }^{21}$ and in $\mathrm{SH}$ mode. ${ }^{12}$ These investigations found typical astrometric precisions of $\sigma \sim 0.4 \mathrm{mas}$, about twice the expected error. There are several reasons to explain the discrepancy, which are discussed in the publications. ${ }^{12,21}$ Unfortunately, the most detailed of these investigations ${ }^{21}$ suffered from instrumental problems due to a miss-calibration of the MAD derotator (fixed for the second SD run), which hampered a more sophisticated characterization of the astrometric properties of the instrument. Nevertheless, the investigation discovered that dithering by moving the detector stage introduced a significant deterioration of the astrometric precision. ${ }^{21}$ Altogether, the 19 scientific publications
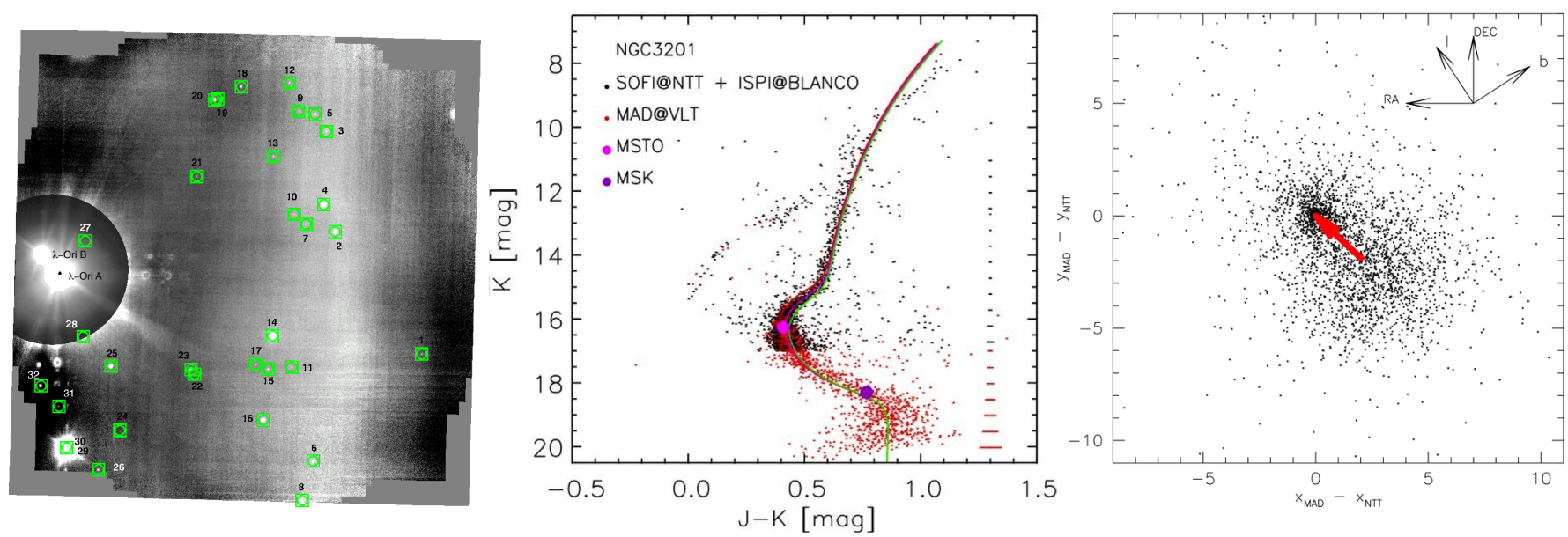

Figure 5. Left: H-band image of $\lambda$-Orionis illustrating the dynamic range of MAD. The levels around the bright binary star $\lambda$-Orionis A have been stretched to enhance the contrast. ${ }^{4}$ Middle: Color-Magnitude diagram of the GC NGC 3201 (the deepest in the near-IR for any GC) showing the photometric precision delivered by MAD. ${ }^{11}$ Right: Proper-motion vector of the old metal-poor Bulge cluster HP 1 in pixel coordinates. In sky coordinates the proper-motion vector is $\vec{\mu}=\left(0.0588^{\prime \prime}, 0.05488^{\prime \prime}\right)$ over a time span of 14.25 years. $^{12}$

based on MAD data have proven that MAD fully met the scientific top-level requirements for a state-of-the-art MCAO imager.

\section{MAD GREATEST HITS: THE MOST CITED PAPERS}

To conclude this presentation we report in this section a more detailed discussion of the results of the highest impact MAD papers (listed in Figure 3). These results are particularly interesting because they represent important contributions to the corresponding fields of Astronomy with exciting new results on topical issues.

\subsection{The universality of the stellar Initial Mass Function (IMF).}

Studies of the field, young clusters and associations, and old globular clusters, suggest that the vast majority were drawn from a universal system IMF: a power law of Salpeter slope $(\Gamma=1.35)$ above a few solar masses, and a log-normal or shallower power-law $(\Gamma \sim 0-0.25)$ for lower mass stars, although the shape and universality of the IMF for very low masses are still under investigation. Figure 6 shows in the left panel the IMFs of some of the best studied clusters and associations in our Galaxy, and in the right panel the combined HST/NTT IMF of the 30 Doradus cluster in the LMC, which is the most accurate IMF for a young massive cluster. ${ }^{22}$ The best fitting power-law slope for 30 Dor, $\Gamma=1.17 \pm 0.06$ (for $M>3 M_{\odot}$ ), is consistent with the Salpeter slope. Most observations are incomplete for masses below $\sim 0.5 M_{\odot}$ (more below) and the new results for 30 Doradus and NGC 3603, two young massive clusters observed by MAD, indicate that our knowledge is also incomplete at the high-mass end of the IMF, previously thought to have a hard physical limit of $\sim 150 \mathrm{M}_{\odot} .{ }^{6}$ 

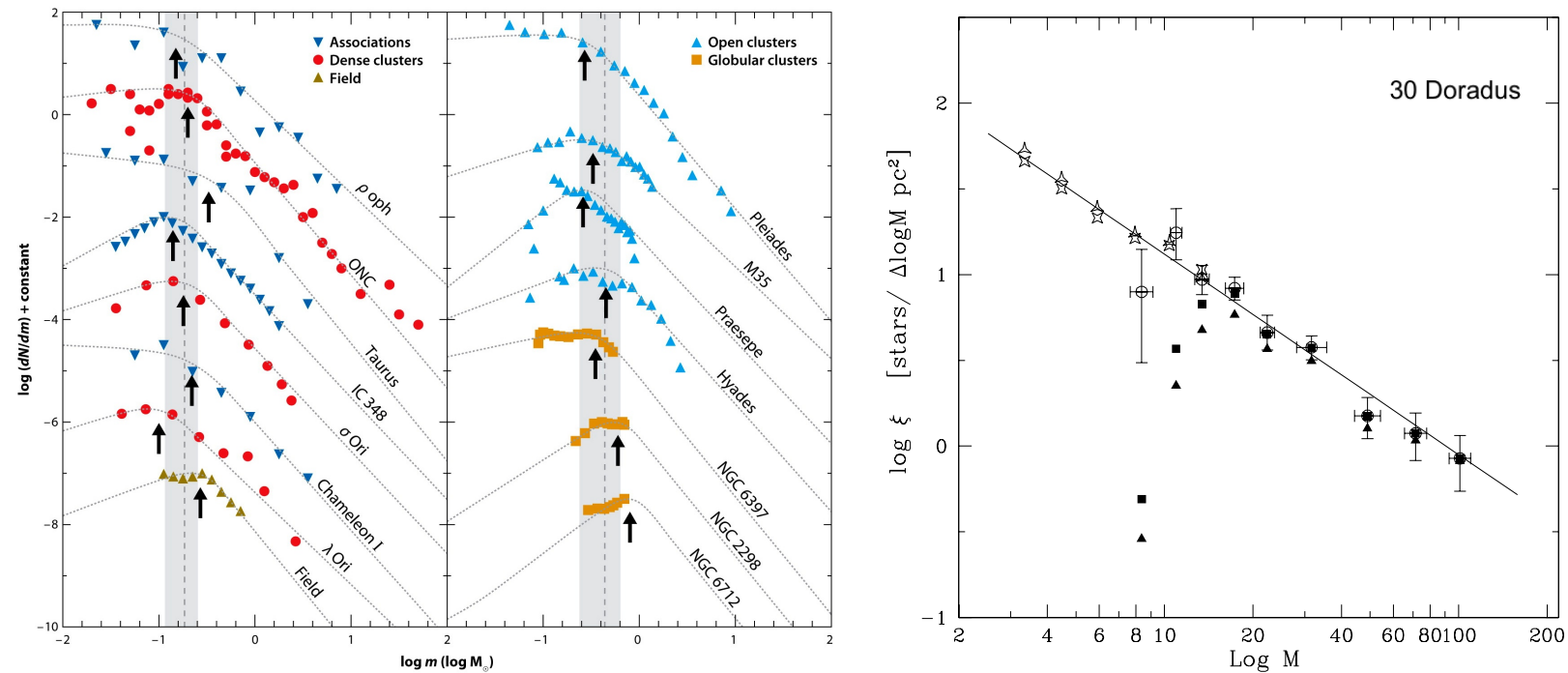

Figure 6. Left: Initial mass function of a number of young Galactic clusters and associations taken from Bastian et al. ${ }^{23}$ The IMF appears to be 'universal' for stellar masses above $\sim 0.5 M_{\odot}$. For lower masses it is difficult to distinguish genuine IMF variations from fluctuations due to incompleteness or small number statistics. Right: IMF of 30 Doradus. This, the best determined IMF for massive stars, does not include stars more massive than $120 M_{\odot}$ because, at the time, theoretical evolutionary tracks for such stars did not exist in the literature. ${ }^{22}$

A particularly thorny issue concerning the stellar IMF is that most studies do not account for binarity, while it is widely accepted that between $50 \%$ and $100 \%$ of all massive stars are in binary or multiple systems. Without knowing the mass distribution of companions, however, it is difficult if not impossible to correct the observed star counts for the effects of multiplicity, so this remains an open question. The other important unknowns, which are particularly relevant for population synthesis studies, are the lower and upper mass limits of the IMF. Below we present a brief discussion of the contribution of MAD to address these crucial issues.

\subsubsection{Multiplicity}

A number of MAD programs were devoted to completing the census of binaries in nearby clusters, in particular $\sigma$-Ori, $\lambda$-Ori, and Trumpler 14 in Carina. ${ }^{3,4,8}$
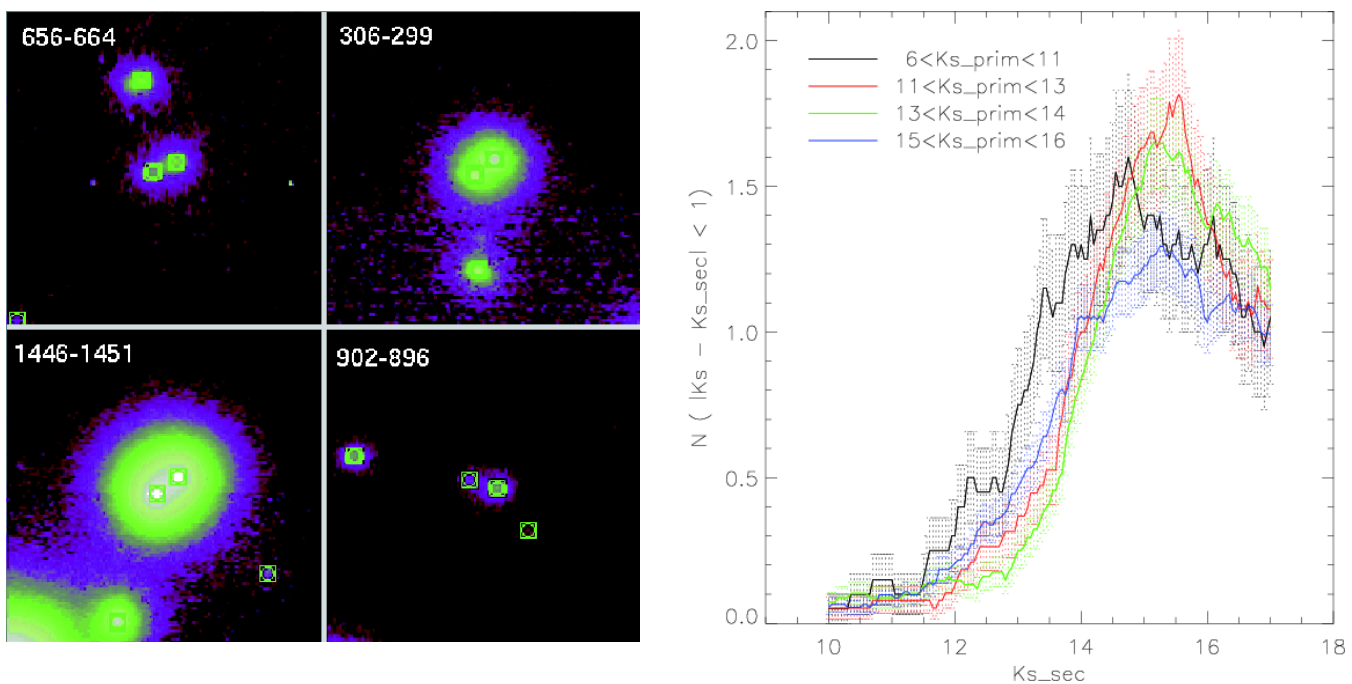

Figure 7. Left: MAD images of close pairs in Trumpler 14. Each box is $6^{\prime \prime} \times 6^{\prime \prime}$ and the pair separations are in the range $0.24^{\prime \prime}-0.30^{\prime \prime} .{ }^{8}$ Right: Average number of companions per star as a function of companion brightness. The colors code the brightnesses of the central stars as shown in the figure. 
Figure 7 illustrates the power of MAD to detect new binaries in nearby clusters. In particular, the combination of acute image quality and large dynamic range allows MAD to probe the presence of very low mass companions to 'single' bright stars down to the sub-stellar regime. The right-panel of Figure 7 shows the mass distribution of secondary stars for different mass ranges of the primaries. Massive stars $\left(M \gtrsim 5 M_{\odot}\right)$ have about twice as many solar-mass companions as lower mass stars, ${ }^{8}$ confirming that indeed a large fraction of massive stars are in multiple systems.

\subsubsection{The upper and lower mass limits of the IMF}

The lower-mass end $-M_{l o w}$

The combination of high Strehl, wide field, uniform PSF, and large dynamic range makes MCAO an ideal tool to study the low mass end of the stellar IMF. This is made difficult by the fact that low-mass stars in young clusters have still not reached the main-sequence, so in addition to the observational burden of observing faint stars in crowded fields, there are theoretical uncertainties that make assigning masses and ages to pre-main sequence stars (PMS) rather uncertain. This is illustrated in Figure 8 that reproduces the faint-end of the MAD color-magnitude diagram of Trumpler $14 .{ }^{24}$ The figure illustrates the commonly observed feature that isochrones of different ages are needed to match the loci of PMS in young clusters. It is still not clear whether this is due to effects such as rotation or disks in PMS stars, or to a real spread in ages. As shown in the figure, these uncertainties affect mostly stars of masses lower than $M \lesssim 0.7 M_{\odot}$, which is the region where the slope of the IMF changes as shown in Figure 6. In Tr 14, the MAD observations found that the IMF bends at approximately the mass expected from observations of other clusters, ${ }^{23}$ but with a shallower slope. Moreover, MAD seems to find an up-turn for $M \lesssim 0.3 M_{\odot},{ }^{24}$ which in the compilations shown above ${ }^{23}$ is only seen in the Pleiades cluster.
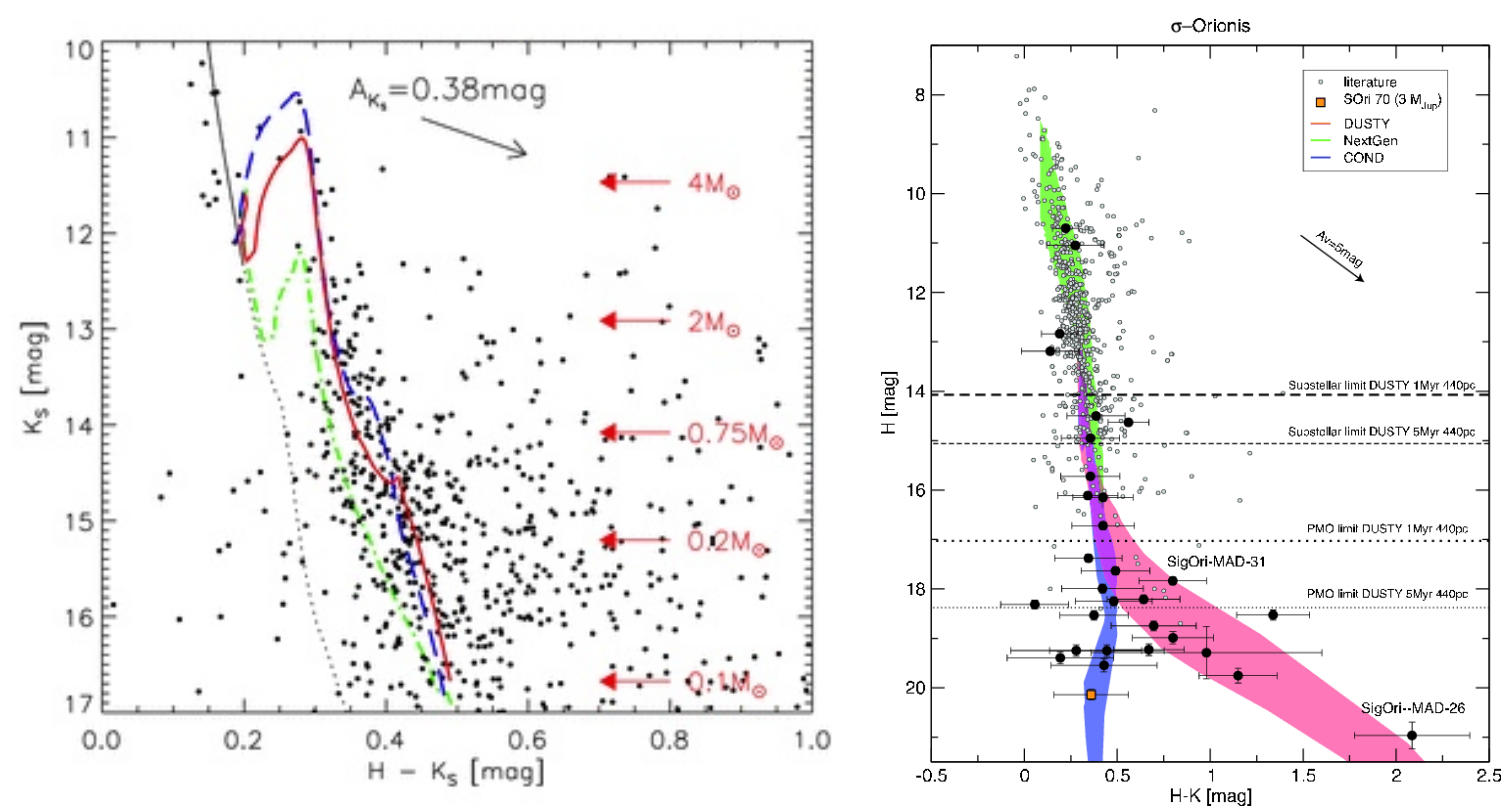

Figure 8. Left: MAD color-magnitude diagram for low-mass stars in Tr. 14. The lines represent PMS isochrones of 0.5, 1.0, and 3 Myr. The labelled masses correspond to the 1 Myr isochrone. ${ }^{24}$ Right: Color-magnitude diagram of the young cluster $\sigma$-Ori. The shaded areas show theoretical isochrones between 1-5 Myr for the different mass regimes. The MAD data are the black dots with error bars. The dashed horizontal lines show the substellar limit for the $1 \mathrm{Myr}$ and $5 \mathrm{Myr}$ isochrones. The dotted horizontal lines show the deuterium-burning (PMO) limit for the same ages. ${ }^{3}$

In nearby young clusters and star-forming regions, near-IR imagers with and without AO can probe well into the sub-stellar regime, habited by brown dwarfs (BDs) and planetary mass objects (PMOs), where the boundary between stars and planets becomes rather blurry. The 'official' lower-mass limit for bona-fide stars is $M \sim 0.07-0.09 M_{\odot}$; lower mass stars being unable to burn hydrogen in their cores. Brown dwarfs are thought to burn deuterium or lithium, which probably ignite for masses as low as $M \simeq 0.01 M_{\odot}$. The right panel of 
Figure 8 shows the color-magnitude diagram of the $\sigma$-Ori cluster obtained combining MAD data with data from the literature. The figure shows that MAD probes well into the PMO regime, as indicated by the dotted lines in the figure, thus allowing to probe the cluster stars for sub-stellar and planetary mass companions. ${ }^{3}$ The MAD observations also detected a significant number of free-floating PMOs, which are considered to form differently from normal stars. Again, these free floating low-mass objects complicate the empirical definition of the low-mass limit of the IMF.

The upper-mass end $-M_{u p}$

Until very recently it was widely hypothesized that stars more massive than $\sim 150 M_{\odot}$ could not form. The observational evidence for this hypothesis stems from star counts in young massive clusters (YMC) having masses larger than $\gtrsim 10^{4} M_{\odot}$, where statistically one expected to find several stars more massive than $150 M_{\odot}$ whereas none had ever been found. In fact, the belief that ultra-massive stars could not form due to physical reasons was so entrenched, that grids of evolutionary models (isochrones) for stars more massive than $150 M_{\odot}$ had never been published. So, although observations of several YMCs (e.g. Arches; 30 Dor; NGC3603; etc.) hinted at the existence of ultra-massive stars, no isochrones were available to weigh them.

This paradigm changed in 2010 thanks to observations with HST and VLT including MAD. Figure 9 reproduces MAD images of central regions of 30 Dor and NGC 3603, which show that the central most luminous 'star' in each cluster is actually resolved into several components (called $a 1, a 2, a 3$ for 30 Dor and $A 1 a, A 1 b$ for NGC 3603). Thus, the most luminous stars in these clusters R136a and NGC3603A1 are in fact resolved into these components.
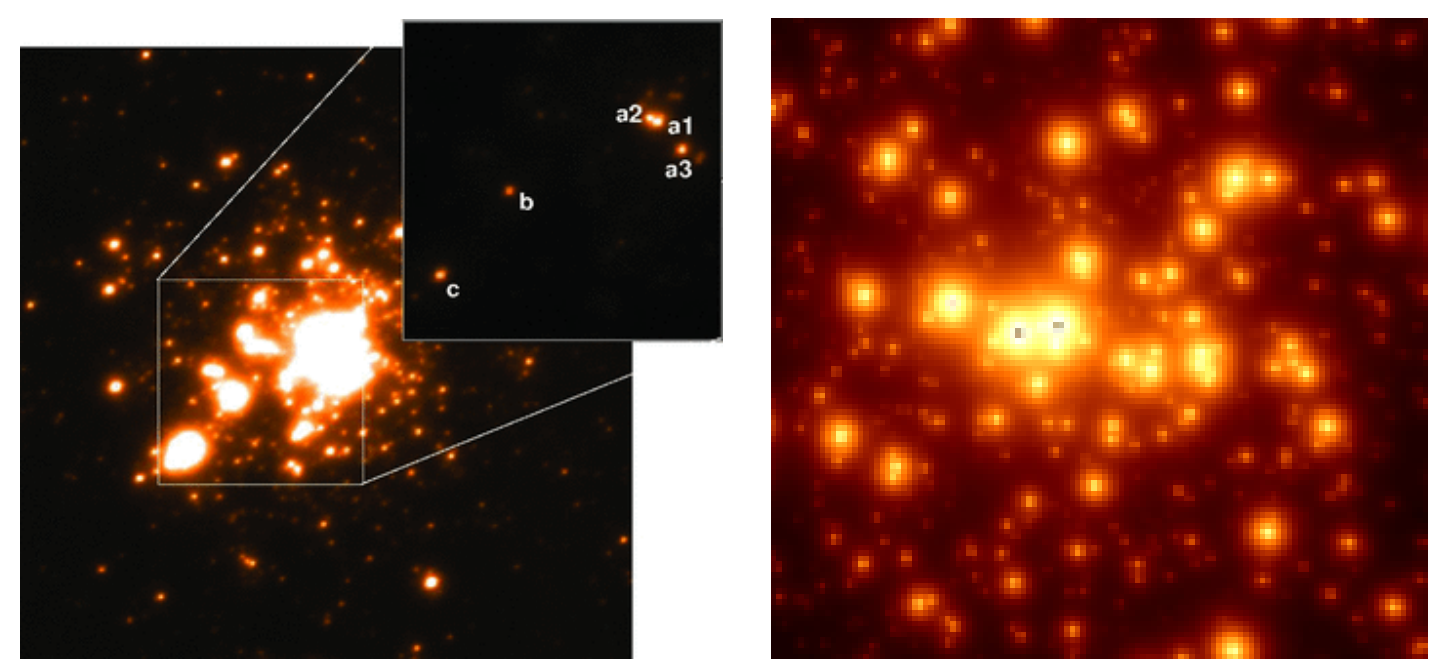

Figure 9. Left: MAD $K_{s}$-band image of the core of the 30 Dor cluster. The insert shows the three components of R136a, once thought to be a single star. ${ }^{5}$ Right: MAD image of the central part of the massive Galactic cluster NGC 3603 (unpublished image courtesy of Joao Alves). Star A1 is resolved into two components, A1a and A1b, of which only A1a is a known binary.

The spectral-energy distributions (SED) of the 30 Dor stars indicated temperatures well in excess of $50,000^{\circ} \mathrm{K}$ and more than $40,000^{\circ} \mathrm{K}$ for NGC $3603 .{ }^{6}$ This suggested that these stars (and also stars $c$ in 30 Dor and $B$ and $C$ in NGC 3603) could be extremely massive. Thus, Crowther et al. ${ }^{6}$ computed a new grid of isochrones using the Geneva code, which includes the effects of (fast in this case) rotation, for initial masses between $85 M_{\odot}$ and $500 M_{\odot}$. The results are reproduced in Figure 10, which shows that indeed, if these stars are not binaries, the four stars in 30Dor and two in NGC 3603 probably had initial masses close or above the canonical $150 M_{\odot}$ limit. In fact, for the most luminous of these stars, R136a1, Crowther et al. ${ }^{6}$ infer a present day mass (PDM) of $\sim 265 M_{\odot}$ and an initial mass of $\sim 320 M_{\odot}$ with estimated $1 \sigma$ errors of $(+100,-40) M_{\odot}$.

We remark, however, that the issue of binarity of these hyper-giant stars is not completely resolved. NGC 3603A1 and NGC 3603C are confirmed binaries and 30 Dor-c is also a probable binary, while 30 Dor-B, R136a1 ,a2, and 

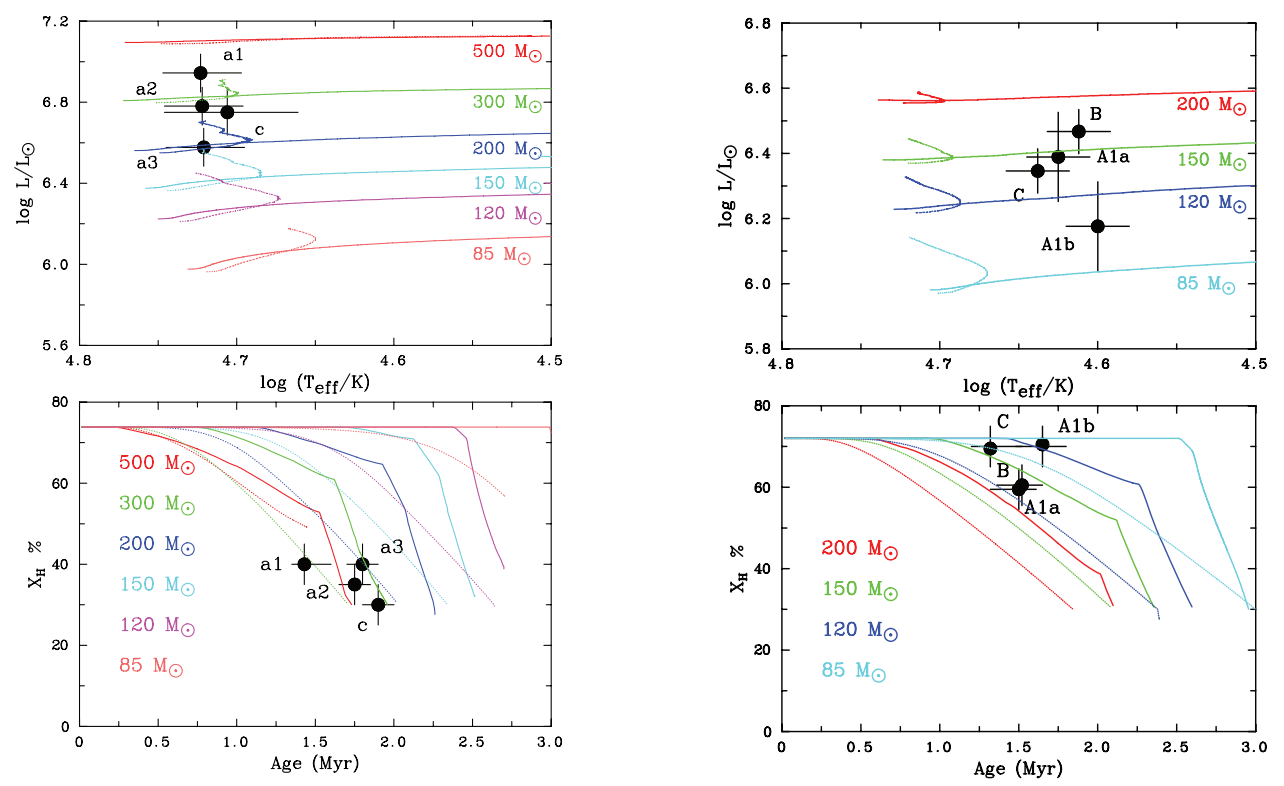

Figure 10. Geneva isochrones for initially rotating (thin lines) and non-rotating (thick lines) stars of initial masses up to $500 M_{\odot}$ compared to the observations of 30 Doradus (left) and NGC 3603 (right).

a3 are presumed to be single on the basis of their observed X-ray luminosities and the lack of detected radial velocity variations.

The statistics for both clusters are consistent with an upper mass limit of $M_{u p} \sim 300 M_{\odot}$. In other words, in order to detect stars more massive than this limit one would need to observe YMCs substantially more massive than 30 Dor $\left(\sim 10^{5} M_{\odot}\right)$, no examples of which are known in the Local universe.

\subsubsection{Summary}

MAD contributed to advance current knowledge about the stellar IMF, and hence about the physics of starformation. Much remains to be done, specially at both ends of the stellar mass spectrum. At the low-mass end, MAD demonstrated the potential of MCAO instruments on large telescopes to obtain complete censuses of the populations of young clusters down to the sub-stellar mass regime. At the high-mass end, MAD resolved the most massive stars in the dense cores of young massive clusters and contributed to the tantalizing suggestion that some of these stars may be hypergiants of several hundred solar masses. The Extremely Large Telescopes (ELTs) of the future, equipped with state-of-the-art MCAO imagers, should be able to resolve the stellar populations of YMCs beyond the mass of 30 Dor and thus to find (or not) stars even more massive than R136a1.

\subsection{Our Galaxy - the Milky-Way - as cosmological probe}

Understanding the assembly history of the Milky-Way galaxy (MW) and its satellites has spawned the socalled near-field cosmology, which has already contributed to refine galaxy-formation models within the $\Lambda$ CDM paradigm. In particular, the observation that our Galaxy contains too many luminous satellites, and far too few low luminosity ones, compared to the number of dark-matter halos predicted by CDM models, has led to refinements of the semi-analytic treatment of the evolution of baryons inside dark-matter halos. Also, the observed mass-density profiles of the best studied satellites do not resemble the cuspy dark-matter halos predicted by CDM. Again, refinements in the treatment of baryonic physics has allowed to find ways out of this problem. ${ }^{25}$

A critical and as yet unsolved problem is the flat (pancake-like) distribution of the satellites of the MW. This is illustrated in Figure 11 that shows that the MW satellites are concentrated in a highly flattened structure almost perpendicular to the Galactic plane, recently baptized the Vast Polar Structure (VPOS). ${ }^{26}$ The discovery of Globular Clusters (GCs) with multiple stellar populations brings new ingredients to our understanding of the formation history of the Milky-Way. 
The majority of GCs have rather uniform iron-abundances, and those showing multiple populations have chemical enrichment patterns indicative of self-pollution. However, the most massive clusters (e.g. $\omega$-Cen and M54) also show a significant spread in iron abundance, similar to that observed in dwarf galaxies and in the Galactic bulge. The most likely explanation is that these GCs are the remnant cores of satellite galaxies that have been tidally disrupted in the process of being accreted by the Milky-Way. In fact, several of the stellar streams in the MW, which are the remnants of tidally-destroyed MW satellite, are also flattened along the VPOS. ${ }^{26}$
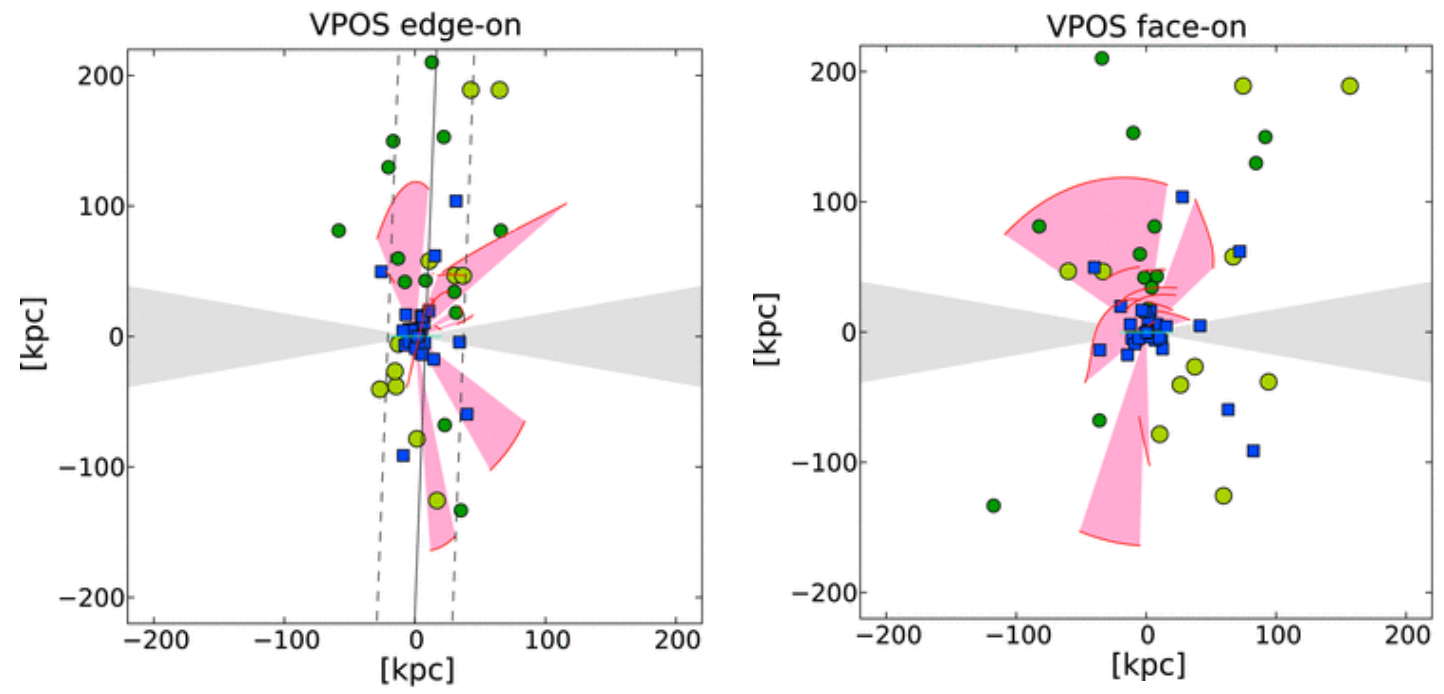

Figure 11. Plane of satellites of the Milky-Way. The 11 classical satellites are shown as large (green) dots, the 13 new satellites are represented by the smaller (green) dots, and young-halo GCs are plotted as (blue) squares. The (light-red) shaded regions illustrate the planes defined by the stellar streams of dwarf galaxies tidally disrupted by the Milky-Way magnified by a factor of 3 to ease the comparison. $\pm 10^{\circ}$ of the MW disc is shown by the horizontal grey areas. The satellite galaxies and young-halo GCs are seen to be well aligned in the edge-on view (left panel), while the face-on view (right panel) shows that the planes of most streams are also aligned with the $\operatorname{VPOS}^{26}$ (A movie rotating this view over $360^{\circ}$ can be found online at http://www. astro.uni-bonn.de/download/.

With this background it seems natural to imagine that the building blocks of the Galactic bulge may have been satellites accreted by the young MW, and that some of the Bulge GCs could, indeed, be the remnants of these building blocks. Observationally, however, disentangling the populations of Bulge GCs from the field is hindered by differential foreground extinction, problem that is substantially alleviated at IR wavelengths where the dust extinction is much lower.

Thus, several MAD-SD programs aimed at studying the stellar populations of Bulge GCs, and in particular of the most massive of these clusters, Terzan 5, which contains about $25 \%$ of the milli-second pulsars known in Galactic GCs. ${ }^{10}$ The excellent MAD data revealed a surprising (but in hindsight not unexpected) result: Figure 12 shows in the left panel the amazing MAD K-band image of the cluster, which clearly illustrates the difficulties of doing photometry in Bulge fields. The right panel shows the color-magnitude diagram of the cluster obtained combining the MAD and archival HST/ACS data (corrected for differential extinction using the MAD $J$ - and $K$-band data). The unexpected double horizontal branch (HB) of Terzan 5 is clearly seen in this figure revealing, for the first time, multiple stellar population in this cluster.

Keck spectroscopy of a number of HB stars in each group showed that the two populations have quite different iron abundances. ${ }^{10,14}$ The best isochrone fits to the two HB populations (showed in the figure) also indicate different helium abundances $(Y)$ and different ages $(t)$ : a young 6 Gyr-old metal-rich population, and an old (12 Gyr) metal-poor component. ${ }^{10}$ The spread in metallicity and the chemical enrichment patterns of Ter 5 are so similar to those of the Bulge field stars that it is almost inevitable to contemplate a common origin. Moreover, the MAD observations also revealed that Ter 5 is significantly more massive and less concentrated than previously thought. ${ }^{13}$ In fact, with an estimated mass of $M_{T} \sim 2 \times 10^{6} M_{\odot}$, Ter 5 is one of the most massive 
GCs in the MW. Moreover, the iron enrichment and the spatial segregation of the younger population indicate that the cluster must have been substantially more massive in the past (in order to retain the iron-enriched material ejected at high velocities by supernova explosions). ${ }^{13}$

The dwarf satellites of the Milky-Way have typical masses of $10^{7} M_{\odot}$ and had at least ten times higher initial masses. Therefore, it can be argued that Ter 5 is the remnant core of a dwarf galaxy that was accreted onto the Galactic bulge losing most of its stars in the process. The ejected stars would now be found in the field, thus accounting for the observed similarities in iron-abundance and chemical enrichment patterns between Ter 5 and the Bulge field populations. These observations favor the scenario where the Galactic bulge evolved from an initial disk/bar and its interactions with other sub-structures. In this scenario, Ter 5 would be the remnant of one of the building-blocks of the bulge of the MW. This is actually consistent with the MAD observations of the metal-poor Bulge GC HP 1 already mentioned in Section 2.3. ${ }^{12}$
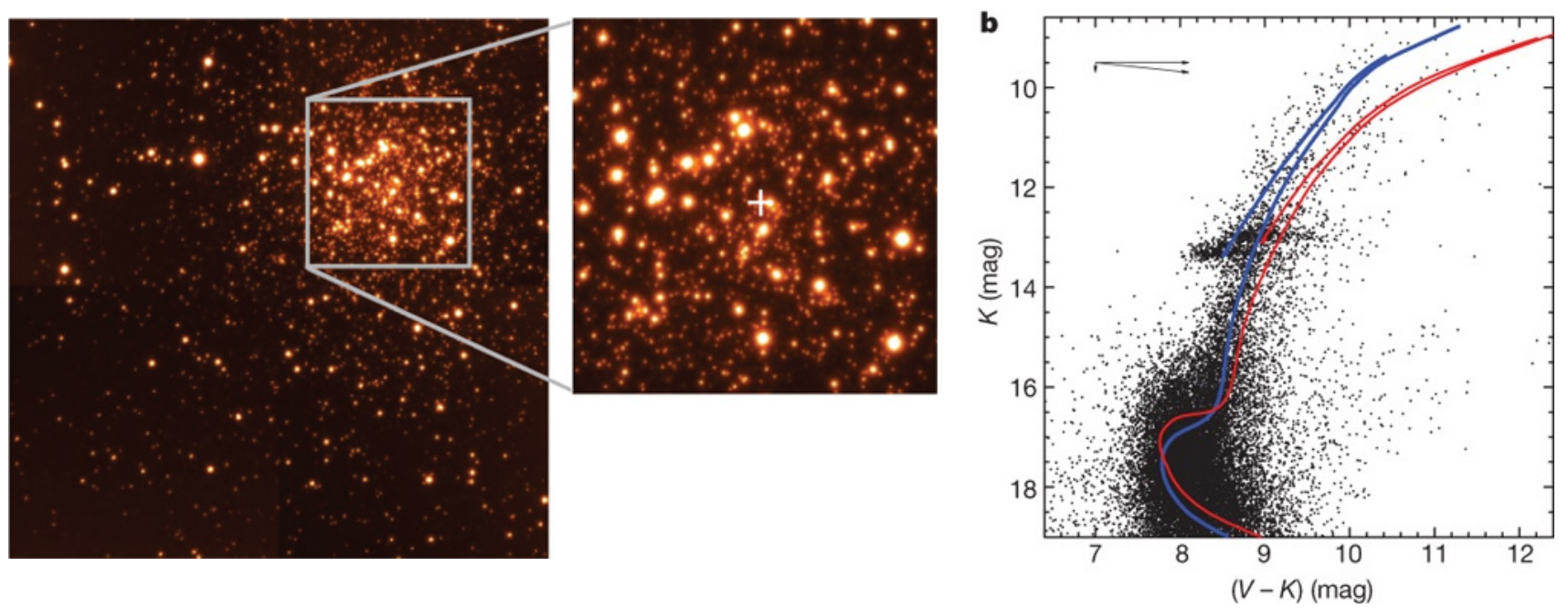

Figure 12. Left: MAD $K$-band image of the central region of Terzan 5 . The Strehl ratios range from $15 \%$ to $24 \%$ over the entire - almost diffraction-limited $\left(\mathrm{FWHM}=0.1^{\prime \prime}\right)$ - image. Right: Combined HST/ACS and VLT/MAD colormagnitude diagram of Ter 5 clearly showing the two populations of different iron abundance detected in this cluster. The effect of reddening in magnitude and color is indicated by the two arrows at the top of the diagram. The ACS V-band photometry was corrected for differential reddening using the MAD JK data. The isochrones correspond to $(Z, Y, t)=(0.01,0.26,12 G y r)$ for the old metal-poor component (blue) and $(0.03,0.29,6 G y r)$ for the young metal-rich component (red), where $Z$ is the iron abundance in metallicity units, $Y$ is the helium abundance, and $t$ is the age of the isochrone.

\subsubsection{Summary}

The discovery of multiple populations in massive globular clusters is beginning to change our ideas about the formation of the Milky-Way galaxy, and thus about galaxy formation in general. MAD demonstrated how nearIR observations with MCAO instruments can further revolutionize the field. Future observations of the stellar populations in the bulge and the clusters in M31 with ELTs, for example, would be crucial to understand whether the MW is a Black-Swan, or whether it formed the way most spiral galaxies do.

\section{CONCLUSIONS}

In its short telescopial life, MAD produced a surprising number of novel scientific results thus heralding the era when very-large $(>8 \mathrm{~m})$ and extremely-large $(>25 \mathrm{~m})$ telescopes equipped with wide-field MCAO imagers will scout the Universe searching for answers to the most fundamental questions of Astronomy. ${ }^{17}$ MAD passed its scientific design goals with flying colors attesting, once again, to the power of human ingenuity. 


\section{ACKNOWLEDGMENTS}

MAD Science Demonstration observations were done in a-fortiori service mode, which required intensive communications between the MAD team and the PI's of the programs. We would like to thank all PI's for their excellent disposition to help in the preparation of the observations, and to congratulate them for the amazing quality of the papers they produced.

\section{REFERENCES}

[1] Marchetti, E. et al., "MAD on sky results in star oriented mode," Proc. SPIE 7015, 70150F-1 (2008).

[2] Arcidiacono, C. et al., "An update of the on-sky performance of the Layer-Oriented wave-front sensor for MAD," Proc. SPIE 7736, 77363D-1 (2010).

[3] Bouy, H. et al., "A deep look into the cores of young cluster. I. $\sigma$-Orionis," Astron. Astrophys. 493, pp. 931-946 (2009).

[4] Bouy, H. et al., "A deep look into the cores of young cluster. II. $\lambda$-Orionis," Astron. Astrophys. 504, pp. 199-209 (2009).

[5] Campbell, M. A. et al., "VLT-MAD observations of the core of 30 Doradus," Mon. Not. R. Atron. Soc. 405, pp. 421-435 (2010).

[6] Crowther, P. A. et al., "The R136 star cluster hosts several stars whose individual masses greatly exceed the accepted $150 \mathrm{M}_{\odot}$ stellar mass limit," Mon. Not. R. Atron. Soc. 408, pp. 731-751 (2010).

[7] Bouy, H., Kolb, J., Marchetti, E., Martin, E., Huélamo, N., and Barrado y Navascues, D., "Multi-conjugate adaptive optics images of the Trapezium cluster," Astron. Astrophys. 477, pp. 681-690 (2009).

[8] Sana, H. et al., "A MAD view of Trumpler 14," Astron. Astrophys. 515, A26 (2010).

[9] Moretti, A. et al., "MCAO near-IR photometry of the globular cluster NGC 6388: MAD observations in crowded fields," Astron. Astrophys. 493, pp. 539-546 (2009).

[10] Ferraro, F. et al., "The cluster Terzan 5 as a remnant of a primordial building block of the galactic bulge," Nature 462, pp. 483-486 (2009).

[11] Bono, G. et al., "ON A NEW NEAR-INFRARED METHOD TO ESTIMATE THE ABSOLUTE AGES OF STAR CLUSTERS: NGC 3201 AS A FIRST TEST CASE," Atrophys. Journ. Letters 708, L74-L79 (2010).

[12] Ortolani, S. et al., "A FOSSIL BULGE GLOBULAR CLUSTER REVEALED BY VERY LARGE TELESCOPE MULTI-CONJUGATE ADAPTIVE OPTICS," Astrophys. Journ. 737, pp. 31-40 (2011).

[13] Lanzoni, B. et al., "New density profile and structural parameters of the complex stellar system terzan 5," Astrophys. Journ. 717, pp. 653-657 (2010).

[14] Origlia, L. et al., "SPECTROSCOPY UNVEILS THE COMPLEX NATURE OF TERZAN 5," Astrophys. Journ. Letters 726, L20-L24 (2010).

[15] Momany, Y., Ortolani, S., Bonatto, C., Bica, E., and Barbuy, B., "Multi-Conjugate Adaptive VLT imaging of the distant open cluster FSR 1415," Mon. Not. R. Atron. Soc. 391, pp. 1650-1658 (2008).

[16] Gullieuszik, M. et al., "Resolving stellar populations outside the Local Group: MAD observations of UKS 2323-326," Astron. Astrophys. 483, L5-L8 (2008).

[17] Fiorentino, G., Tolstoy, E., Diolatti, E., Valenti, E., Cignoni, M., and Mackey, A., "MAD about the Large Magellanic Cloud: Preparing for the era of Extremely Large Telescopes," Astron. Astrophys. 535, A63 (2011).

[18] Falomo, R. et al., "The jet of the BL Lacertae object PKS 0521-365 in the near-IR: MAD adaptive optics observations," Astron. Astrophys. 501, pp. 907-914 (2009).

[19] Liuzzo, E. et al., "The jet of the BL Lacertae object PKS 2201+044: MAD near-IR adaptive optics observations and comparison with optical, radio and X-ray data," Astron. Astrophys. 528, A36 (2011).

[20] Wong, M. H., Marchis, F., Marchetti, E., Amico, P., Tordo, S., Bouy, H., and de Pater, I., "A shift in Jupiters equatorial haze distribution imaged with the Multi-Conjugate Adaptive Optics Demonstrator at the VLT," Bulletin of the American Astronomical Society 41, p. 56 (2009).

[21] Meyer, E., Kürster, M., Arcidiacono, C., and Rix, H.-W., "Astrometry with the MCAO instrrument MAD. An analysis of single-epoch data obtained in the layer-oriented mode," Astron. Astrophys. 532, A16 (2011). 
[22] Selman, F., Melnick, J., Bosch, G., and Terlevich, R., "The ionizing cluster of 30 Doradus. III. Star-formation history and initial mass function," Astron. Astrophys. 347, 532 (1999).

[23] Bastian, N., Covey, K. R., and Meyer, M. R., "A Universal Stellar Initial Mass Function? A Critical Look at Variations," Ann. Revs. Astron. Astrophys. 48, 339-389 (2010).

[24] Rochau, B. et al., "A benchmark for multi-conjugated AO: VLT-MAD observations of the young massive cluster Trumpler 14," Mon. Not. R. Atron. Soc. 418, 949-961 (2011).

[25] Parry, O. H., Eke, V. R., Frenk, C. S., and Okamoto, T., "The baryons in the Milky Way satellites," Mon. Not. R. Atron. Soc. 419, 3304-3318 (2012).

[26] Pawlowski, M. S., Pflamm-Altenburg, J., and Kroupa, P., "The VPOS: a vast polar structure of satellite galaxies, globular clusters and streams around the Milky Way," Mon. Not. R. Atron. Soc. 423, 1109 (2012). 